\title{
ESTIMATING PERFORMANCE CHARACTERISTICS THROUGH OBSERVED NASH EQUILIBRIA
}

\author{
KJETIL K. HAUGEN
}

\begin{abstract}
This article uses simple game models to investigate penalty kick shootouts in soccer. As opposed to existing research, which mainly focuses on empirical/experimental problems, the game itself is the main focus here.

Interesting findings include a proposed Nash equilibrium invariance for possible preference differences between penalty kick Executors and Keepers as well as identification and demonstration of how game models may be used in order to estimate player performance characteristics through observed Nash equilibria outcomes.

A non-mixed strategy Nash equilibrium is also shown to be possible with potential interesting consequences for goal keepers.
\end{abstract}

\section{The PENALTy KICK}

The penalty kick in soccer is both the most decisive as well as the simplest subgame arising in European football (soccer). The reason for its simplicity (in game theoretic terms) is the limited number of players $\left(\mathrm{two}^{1}\right)$ as well as a relatively small and well defined strategy space. Its decisiveness is also easy to accept, bearing in mind a significant number of decisive penalty-kick shoot-outs both in previous World and European championships. The famous David Beckham miss in the EURO 2004 quarter final against Portugal may stand as a good example of match outcome importance.

The sports economic relevance of the penalty kick may of course also be questioned. The impact of the penalty kick on relevant sports economic variables such as uncertainty of outcome, fan demand or player wages is perhaps limited. However, its potential importance in match outcome and (most certainly) its game theoretical potential makes it a relevant example of applied game theory in sports economic theory. This view is supported by the fact that very serious scientific publishing channels such as "The American Economic Review" [1] and "Econometrica" [6], [7] have published papers on the topic. However, it seems pointless trying to hide the fact that the practical importance and relevance may be far more obvious in a tactical or coaching perspective than in a descriptive sports economic context.

\footnotetext{
$M S C$ (2010): primary 91A05, 91A 80.

Keywords: sports strategy, sports economics, game theory, penalty kick.

${ }^{1}$ The number of players in a penalty kick can obviously be a matter of discussion. The referee, the audience, the coaches, media and so on may surely have decisive impact on strategic choices and player performance. However, in order to keep complexity at a reasonable level, two-players are assumed.
} 


\section{Literature}

As briefly indicated above, several economists have shown considerable interest in analysing penalty kicks. Especially, I. Palacias-Huerta [6], [7], [8], who in a series of relatively recent papers has treated the concept of mixed strategy Nash equilibria in penalty kicks mainly within an empirical/experimental setting. Among other interesting results, Palacias-Huerta claims that professional players do randomize, and randomize well.

Other types of penalty kick research are related more to the psychological effects/stress involved in taking penalties in decisive matches [9], [2], [3], or focusing more on achievement of some kind of optimal/technical performance strategies [10].

\section{Outhine}

In the following sections, a model alternative to that of Chiappori et al [1] and Palacias-Huerta [6], [7], [8], mainly captured from Haugen [5], will be presented. The main difference between Haugen's and the other models is the simplicity; in the sense that the strategy space is at an absolute minimum. This opens up for some more game theoretic judgements and this paper will hence focus more on the game theoretic aspects of the penalty kick than on the empirical/experimental parts.

Firstly, the basic model is outlined, then the mixed strategy Nash equilibrium is established, and then finally, the paper returns to some "reverse engineering" empirical questions on how such game models may be used in a practical setting. At the very end, a discussion on the possibility of extending these concepts into more advanced game modelling will be conducted, among other aspects involving incomplete and asymmetric information.

\section{THE BASIC MODEL}

The following assumptions are made:

i) The game includes two players, Executor and Keeper ${ }^{2}$.

ii) Both the Executor and the Keeper must make all decisions simultaneously before the penalty kick ${ }^{3}$.

iii) The strategy space is simplified (perhaps oversimplified) to two possible decisions; a middle shot $M$, or a wide shot $W$. Both players may choose from this strategy set only ${ }^{4}$.

\footnotetext{
${ }^{2}$ This may, as indicated in Section 1, be a questionable assumption. Especially, fans at the match may in certain cases play an important role in explaining the outcome of penalty kicks. However, this assumption is kept, primarily for simplicity, but also in accordance with the existing research cited.

${ }^{3}$ This assumption is probably even more questionable than the previous one, especially if the 1997 FIFA penalty kick rule change is considered. According to [4], this rule change implied the possibility for Keeper movement (along the line though) before the penalty execution. Surely, this opens up for sequential actions both for the Keeper, but also for the Executor, making the classical result of unique mixed strategy equilibria questionable

${ }^{4}$ This assumption sums up to a dual choice for both players, the Executor could either shoot (deliberately) in the middle $(M)$ or wide $(W)$. That is, all possible wide shots, left, right high low, hard soft etc. are summed up in the single decision variable $W$. If the Keeper chooses $W$,
} 
iv) The Executor always hits the goal if he chooses his strategy $M$. However, if strategy $W$ is applied, a probability $q$ of missing the goal is introduced.

v) If both the Keeper end the Executor chooses the $M$ strategy, all shots are saved $^{5}$.

vi) If the Keeper and the Executor choose different strategies, all shots yield a goal ${ }^{6}$.

vii) If both players choose the $W$ strategy, some shots are saved (basically if the Keeper chooses to throw himself in the same direction as the shot), and some yield a goal - if the opposite occurs.

ix) The probability of a goal hence exists, and is denoted $p$.

$\mathrm{x})$ The utility of a goal or no goal is assumed symmetric as outlined in Table 1 and the players obey Von-Neuman-Morgenstern utility theory ${ }^{7}$.

\begin{tabular}{|l|c|c|}
\hline & GOAL & NO GOAL \\
\hline EXECUTOR & 1 & 0 \\
KEEPER & 0 & 1 \\
\hline
\end{tabular}

Table 1. Utilities for each player in the Penalty kick game.

xi) All information above is visible and known to both players before the penalty kick (a game of complete information).

For obvious reasons, it should be noted that the modelling frame outlined through assumption i) to xi) fits a penalty shoot-out far better than an ordinary in-game penalty kick. This seems reasonable by two arguments. Firstly, because a penalty shoot-out is significantly simpler - there is only one attempt. An ordinary penalty opens up for rebounds by the Executor, as well as other players on the team, greatly enhancing the complexity of the game. Secondly, a shoot-out is (normally) significantly more decisive, putting more pressure on the Executor (primarily) and hence probably makes our assumption on simultaneity more realistic.

Given assumptions i) to xi) above, it is straightforward to construct a pay-off matrix for the two-player simultaneous complete information penalty kick game as shown in Figure 1.

Using the notation $(X, Y)$ representing the Executor choosing action $X$ and the Keeper choosing $Y$, it is straightforward to explain the contents of Figure 1 . In the $(M, M)$ case, all shots are saved (by assumption v)) and hence a certain utility

he decides to go (throw himself) in any direction. A choice of $M$ for the Keeper implies standing still in the middle, awaiting the shot and possibly reacting to it after observing the angle and speed of the ball.

${ }^{5}$ The tiny probability of actually achieving a goal in this situation is omitted for simplicity reasons

${ }^{6}$ The tiny probability of missing the goal in this situation is also omitted (for the time being) by an argument similar to the previous footnote.

${ }^{7}$ For practical purposes, this implies acceptance of the expected utility maximization hypothesis. Note also that this assumption opens up for a direct substitution between expected utilities and probabilities as $E_{U}$ (Executor $)=P(\mathrm{GOAL}) \cdot 1+(1-P(\mathrm{GOAL}) \cdot 0=P(\mathrm{GOAL})$. We will discuss relaxations of this assumption later. 


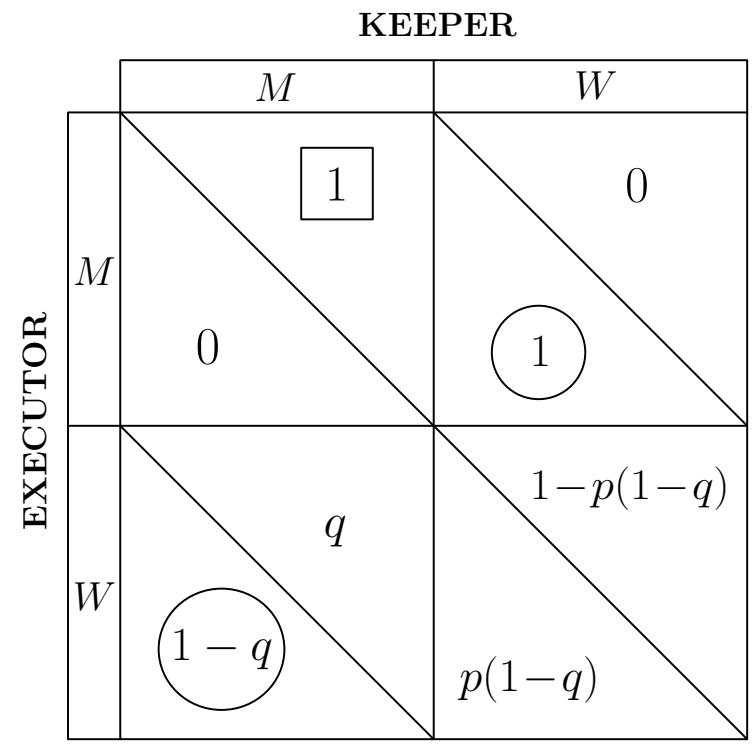

Figure 1. The penalty kick game in normal form.

of 1 for the Keeper and 0 for the Executor. In the $(M, W)$ case, assumption vi) secures a certain goal, and hence utility 1 for the Executor and 0 for the Keeper. In the $(W, M)$ case, a sure goal is the case unless the Executor misses the goal (assumption iv)), the probability of a hit is $(1-q)$ and that of a miss is $q$, hence the observed pay-off in Figure 1. Finally, in the $(W, W)$ case, two things must happen simultaneously for the realization of a goal. The Keeper must not save the shot (probability $p$ ), and the Executor must hit the goal (probability $(1-q)$ ). The total probability must hence (given an inherent assumption of statistical independence) be $p(1-q)$. Consequently, the probability of a save (or no goal) must be 1 minus the former probability.

\section{Some PRELIMINARY RESUlts}

The natural next step is (obviously) to establish Nash equilibrium characteristics of the game of Figure 1. The Best reply functions ${ }^{8}$ (or mappings) are shown by circles and squares for the Executor and the Keeper respectively.

In order to achieve the best reply functions of Figure 1, a limited (and acceptable) set of assumptions on $p$ and $q$ are necessary.

$p$, is the probability of a goal given the choice of $(W, W)$ for both players. Hence, a $p$ of 0 or 1 means either never goal, or always goal, so the following assumption on $p$ seems natural:

$$
0<p<1
$$

\footnotetext{
${ }^{8} \mathrm{~A}$ best reply function (or mapping in this case) is constructed by maximising pay-off for any player as a function of the opponent's actions.
} 
A similar assumption on $q$ seems also reasonable, as a $q$ of either 0 or 1 would imply always or never hitting the goal respectively. Hence,

$$
0<q<1 \text {. }
$$

Given the above assumptions (5.1) and (5.2), it is straightforward to explain the relevant best replies ${ }^{9}$ of Figure 1. As

$$
1-q>0 \Leftrightarrow q<1
$$

and

as well as

$$
1-p(1-q)>q \Leftrightarrow 1-p>q(1-p) \Leftrightarrow q<1
$$

$$
p(1-q)<1
$$

Now, by the absence of pure strategy Nash equilibria (as observed in Figure 1), the famous proof by John Nash [11] secures a unique Nash equilibrium in mixed strategies of this game.

The intuition of such a solution is also relatively straightforward. It is obvious (again given the assumption of simultaneity) that the Executor would maximise his probability of getting a goal by executing his penalty by choosing strategy $W$ if the Keeper chooses $M$ or the other way round. One first attempt would perhaps (historically) be to vary the shots. However, deterministic variation is (at least in principle) predictable. As such, the only way to secure unpredictability in his actions, is to randomize. Obviously, the Keeper will have to follow up such a strategy in order to maximise his probability of a save. Consequently, an equilibrium outcome in mixed strategies seems very reasonable. The mathematical expression $^{10}$ of this Nash equilibrium is shown in equation (5.4) below:

$$
\left\{r^{*}, s^{*}\right\}=\left\{\frac{1-p(1-q)-q}{2-p(1-q)-q}, \frac{1-p(1-q)}{2-p(1-q)-q}\right\} .
$$

In equation (5.4), $r^{*}$ refers to the probability of the Executor choosing $M$, while $s^{*}$ refers to the same choice for the Keeper (in Nash equilibrium).

\section{Further ANALYsis}

Let us start simplifying (5.4) by the added assumption of $q=0$. That is, all wide shots (The Executor choosing $W$ ) hit the goal. Given this assumption, equation (5.4) still holds, and the special case of $q=0$ can simply be entered into it yielding:

$$
\left\{r^{*}, s^{*}\right\}=\left\{\frac{1-p}{2-p}, \frac{1-p}{2-p}\right\} .
$$

The first thing to observe from equation (6.1) is the obvious fact that both players (Executor and Keeper) randomize equally with respect to middle and wide shots. Let us furthermore observe some empirical data from Chiappori et al [1]

\footnotetext{
${ }^{9}$ Best replies for Executor and Keeper are marked with circles and squares respectively in Figure 1.

${ }^{10}$ The algebra behind the calculation is omitted here as it will be illustrated in a later case see appendix B.
} 
and Bar-Eli et al [3]. Chiappori et al [1] observed 459 penalty kicks over a twoyear and a 3 year period of the French and Italian first leagues respectively. Their results show (among others) that $17.2 \%{ }^{11}$ of observed penalty kicks were aimed at the middle. In contrast, Bar-Eli et al observed 286 penalty kicks (unfortunately not linked to leagues) where $28.7 \%^{12}$ were aimed at the middle. The interesting thing with the (way too simplified ${ }^{13}$ ) model returning equation (6.1) is that we can use a reverse argument based on the empirical observations above and estimate $p$ through the Nash equilibrium. Table 2 shows the results of such an estimation.

\begin{tabular}{|l|l|}
\hline$\frac{1-\hat{p}_{C H}}{2-\hat{p}_{C H}}=0.172$ & $\hat{p}_{C H} \approx 0.792$ \\
$\frac{1-\hat{p}_{B E}}{2-\hat{p}_{B E}}=0.281$ & $\hat{p}_{B E} \approx 0.609$ \\
\hline
\end{tabular}

Table 2. Estimated $p$-values for the Chiappori et al [1] data $\left(\hat{p}_{C H}\right)$, and the Bar-Eli et al [3] $\operatorname{data}\left(\hat{p}_{B E}\right)$.

The interesting point to observe here is the fact that observations on directional proportions of penalty kicks, through simplified game theoretical analysis, can provide skill-information ${ }^{14}$ for various leagues or groups of players. Unfortunately, the data used here does not have the necessary disaggregated level, but still the following can be observed: The French and Italian first leagues of Chiappori et al [1] provide a $p$-value close to $80 \%$ as opposed to the Bar-Eli et al [3] data providing a $p$-value close to $60 \%$. Consequently, if one believes that the model leads to (6.1), the conclusion of significant quality differences between the Executors and Keepers of the 2 datasets can be drawn. A simple interpretation would be that there are $20 \%$ more goals on penalty kicks aimed wide where the Keeper goes for the ball in the Chiappori et al [1] dataset indicating significant penalty kick quality differences either between Executors (positively) or Keepers (negatively).

The fact that the Nash equilibrium outcome of penalty kick games can be applied to derive quality information on the players is in itself interesting. Surely, in order to fine tune such estimates, a higher complexity in the game modelling is needed.

Let us now return to the original model leading to equation (5.4). This slightly more complex model shows significant differences compared to the special case leading to (6.1). It is straightforward to observe that $r^{*}<s^{*}$ as

$$
s^{*}=r^{*}-\frac{q}{2-p(1-q)-q} \text {. }
$$

\footnotetext{
${ }^{11}$ Exactly $\frac{79}{459}$.

${ }^{12}$ Exactly $\frac{82}{286}$.

${ }^{13}$ Surely, this model is extremely oversimplified. The simple fact that it predicts equal randomizing behaviour between Executor and Keeper should indicate this. The data of Chiappori et al [1] as well as Bar-Eli et al [3] shows significant differences in the randomizing pattern of the two players - more on this later on.

${ }^{14}$ Again, our model is way too simple. The $p$-parameter, for instance, does not separate quality between Executor and Keeper. Such a separation can, however, be introduced by a more fine-grained modelling.
} 
Now, by (5.2) and (5.3), $2-(p(1-q)+q)$ is positive. The same holds for $q$. Consequently, $\frac{q}{2-p(1-q)-q}>0$ and $r^{*}<s^{*}$. Let us interpret this result. $r^{*}<s^{*}$ means that the share of penalties aimed at the middle by the Executor is smaller than the proportion of Keeper movement standing still. It is perhaps easiest to explain this result if we look at the limiting case. Now let $q \rightarrow 1$ (recall by (5.2) that a $q=1$ is denied through the initial assumptions). Then

$$
\lim _{q \rightarrow 1}\left\{\frac{1-p(1-q)-q}{2-p(1-q)-q}, \frac{1-p(1-q)}{2-p(1-q)-q}\right\}=\{0,1\} .
$$

The meaning of the limiting expression $(\{0,1\})$ is that the less skilled Executors will tend to shoot more wide while the Keeper will tend more to keep standing still. On the limit (of course), the completely hopeless Executor will always shoot wide (and always miss), while the Keeper will always stand still, saving no shots, but neither letting any goals in. This may seem paradoxical - the worse you are at shooting wide the more you will do it. But here, the "gaming" effect must be considered. Keep in mind that the Keeper knows the (bad) quality of the Executor ${ }^{15}$, and takes advantage of it.

The above facts may (with some good will) be interpreted in the following manner: If a penalty shoot-out is observed, the above model (5.4) indicates that a less skilled penalty kick executor to a larger degree (than the more skilled one) would tend to shot more wide. The consequences will then be that less goals are obtained as opposed to the skilled penalty shooters who tend to shoot less wide. Surely, our model defines $q$ as the ability to hit the goal, and in the original modelling "language", our interpretation is unsharpened. However, it should be evident, than $q$ may just as well be interpreted as a combined skill parameter containing both the ability to hit the goal and the ability to get the ball past the Keeper if the Keeper goes for the ball (i.e. chooses the $W$-strategy). As such, the above results seem reasonable. Most of us have observed penalty shoot-outs where the most skilled Executors (typically) start and in some cases choose the $M$-strategy (of course not always with success). However, as the shoot-out moves on, more unskilled players are forced to try, and in many cases one could observe bad wide shots. In essence, this argument boils down to something like: Only skilled penalty kick Executors choose to deviate from a wide strategy. Surely, this kind of hypothesis is testable, unfortunately, data at the correct aggregation level is not present.

At this point, it will prove worthwhile to revert back to the empirical observations of Chiappori et al [1] as well as Bar-Eli et al [3]. Recall that our two models leading to (5.4) and (6.1) predict $r^{*} \leq s^{*}$. The above cited papers do however indicate a quite different "real-world" behaviour. In fact, both articles show significant difference between $r^{*}$ and $s^{*}$ but in the opposite direction. That is $r^{*} \gg s^{*}$. This seemingly puzzling fact may not be that difficult to explain. Recall that our basic modelling contained many simplifying assumptions and it may be of interest to see if a simple relaxation of certain of these assumptions may produce results closer to real world observations. Indeed, this is the case. It turns out that parts of assumption vi) need to be relaxed - not the $(W, M)$-situation, it seems reasonable

\footnotetext{
${ }^{15}$ Recall assumption xi).
} 
to keep the original structure. On the other hand, the opposite situation $(M, W)$ is somewhat too restrictive on Keeper's abilities. It is surely possible though not very possible that a Keeper going for the ball (choosing his $W$-strategy) may save a shot aimed at the middle - for instance with his legs. given this relaxation, it seems reasonable to add yet another probability say $t$ defined as

$$
t=P(\text { Goal } \mid \text { Executor choose } M \text { and Keeper choose } W) \text {. }
$$

Given the definition of (6.2), $1-t$ denotes the relatively small probability of a "save with the feet" discussed in the previous paragraph. It may seem sensible (at first) to merely extend the original model with probabilities $t, 1-t$, but it turns out that the algebraic complexity becomes unnecessary high. Consequently, the special case of the model leading to (6.1) is used. Given these definitions, Figure 2 shows the revised model.

\section{KEEPER}

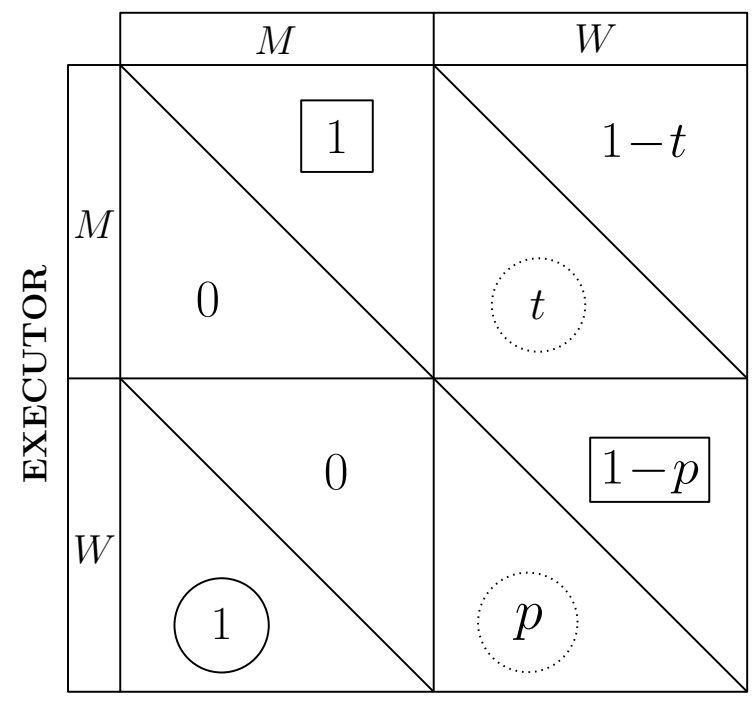

Figure 2. The revised penalty kick game in normal form.

First, observing Figure 2, an important point needs to be stressed. As Figure 2 shows, the original game has changed considerably. Now, the possibility of an added pure Nash equilibrium has emerged ${ }^{16}$. In practice, the added assumption of $t>p$ avoids this possibility, and this is definitely a reasonable assumption. The interpretation is simple. The assumption $t>p$ means that if the Keeper goes (to any side) for the ball, it is easier to get a goal by aiming at the middle than aiming wide.

However, this fact introduces some interesting information on the "nature" of penalty kicks. Surely, for any given $p$, the Keeper can improve his abilities to "save by the feet" by practising. The consequence would imply increasing $1-t$ and hence decreasing $t$. If the Keeper is able to practice enough to reach the limit $t<p$,

\footnotetext{
${ }^{16}$ Observe the dotted circles (best replies) in Figure 2.
} 
the penalty kick will change structurally, from a game with a unique NE in mixed strategies into a game with a unique pure $(W, W)$ NE. This kind of information might be at least principally relevant for goalkeepers and their coaches.

Anyway, let us return to the main point at hand; the derivation of the NE of Figure 2 given an added assumption of $t>p$. The tedious algebra is again omitted, and the reader is again reffered to Appendix B for information on the methodology. The solution is given in equation (6.3):

$$
\left\{r^{*}, s^{*}\right\}=\left\{\frac{1-p}{t-p+1}, \frac{t-p}{t-p+1}\right\} .
$$

Inspecting (6.3), it is evident that the the following holds:

$$
r^{*}>s^{*} \Leftrightarrow 1-p>t-p \Leftrightarrow t<1 .
$$

Consequently, the opposite situation of equations (5.4) and (6.1) is identified if $t<1$. This assumption is also evident, as the alternative $t=1$ would imply the original simplified model (6.1). Thinking somewhat practically should make the sense of this argument. Note that the original two models to a certain extent "underestimated" the abilities of a goalkeeper. The models outlined only opened up for a save in the case of a wide shot. Surely, the new model of Figure 2 opens up some more reality by allowing saves also if shots are aimed at the middle given a choice of $W$ by the Keeper. Obviously, even more realism would enter the model if the possibility of a goal in the $(M, M)$-case was introduced as well as the original $q$-probability. However, the main point with this (still very simplified model) was to establish the existence of mixed NEs where $r^{*}>s^{*}$.

Now, an argument similar to that leading to Table 2 can be applied (though slightly more complex). The following equational system can be used to find estimates for $p\left(\hat{p}_{i}\right)$ and $t\left(\hat{t}_{i}\right)$ by solving

$$
\frac{1-\hat{p}_{i}}{\hat{t}_{i}-\hat{p}_{i}+1}=r_{i}^{*} \text { and } \frac{\hat{t}_{i}-\hat{p}_{i}}{\hat{t}_{i}-\hat{p}_{i}+1}=s_{i}^{*} \forall i \in\{C H, B E\} .
$$

The solution ${ }^{17}$ is:

$$
\hat{p}_{i}=1-\frac{r_{i}^{*}}{1-s_{i}^{*}} \text { and } \hat{t}_{i}=\frac{1-r_{i}^{*}}{1-s_{i}^{*}} .
$$

Table 3 sums up the application of equations (6.5) on the two data sets of Chiappori et al [1] $(\mathrm{CH})$ and Bar-Eli et al [3] $(\mathrm{BE})$.

\begin{tabular}{|c|c|c|c|c|}
\hline & $r_{i}^{*}$ & $s_{i}^{*}$ & $\hat{p}_{i}$ & $\hat{t}_{i}$ \\
\hline \hline$C H$ & $\frac{79}{459}$ & $\frac{11}{459}$ & 0.824 & 0.848 \\
$B E$ & $\frac{82}{286}$ & $\frac{18}{286}$ & 0.694 & 0.761 \\
\hline
\end{tabular}

Table 3. Estimated $p$ - and $t$-values for the Chiappori et al [1], and the Bar-Eli et al [3] data.

First, comparing Table 2 and 3, we observe that the $p$-values differ between the tables. This is of course expected as the two tables originate from two different game models. Second, one observes significant differences between the datasets

${ }^{17}$ Refer to appendix A for the algebra. 
both for $p$ - and $t$-values. Furthermore, the point here is perhaps not to infer too much by this example, but rather to show how this technique can be applied in order to obtain more "quality" information based on the latter model. Still, the interesting point is of course the fact that observed shooting equilibrium strategies $\left(r_{i}^{*}, s_{i}^{*}\right)$, can be used to infer quality information on players. Indeed, it should, hence, be possible to extend the simple modelling frame introduced here even further in order to derive more disaggregated performance information, for instance, separating Executor and Keeper quality.

\section{Preferences of Executors And Keepers}

Certain practitioners might claim that the type of preference structure applied in this article (see Table 1) is unrealistic. A typical argument would go something like this:

"Keepers and Executors face different games in a penalty shoot-out. The Keeper is not expected to save, he will be the hero if a save is the outcome. The Executor, on the other hand, is expected to score on any penalty so he will the scapegoat in the case of a miss".

Surely, such an argument seems reasonable. The interesting question in this setting is whether it makes any difference regarding the Nash equilibrium outcome. The following proposition (under relatively strict assumptions) shows that it does not.

Proposition. Given assumptions i) to xi) and the added assumption of $q=0$, a generalized preference difference between executor and Keeper as defined by Table 4 does not change the Nash equilibrium outcome of the game.

The proof for this proposition is left for Appendix B.

Table 4 defines the generalized preference differences between Keeper and Executor.

\begin{tabular}{|l|c|c|}
\hline & $G O A L$ & $N O G O A L$ \\
\hline EXECUTOR & 1 & 0 \\
KEEPER & $1-\mu$ & $1+\omega$ \\
\hline
\end{tabular}

Table 4. Generalized utilities for each player in the Penalty kick game; $(0<\mu \leq 1$ and $\omega \geq 0)$.

It is readily observed from Table 4 and the range definitions of $\mu$ and $\omega$ in the caption of the table that the following reasonable assumptions then hold $\left(U_{K}\right.$ and $U_{E}$ denote utilities for Keeper and Executor respectively):

$$
\begin{aligned}
& U_{K}(\mathrm{GOAL}) \leq U_{E}(\mathrm{GOAL}), \\
& U_{K}(\mathrm{NO} \text { GOAL }) \geq U_{E}(\mathrm{GOAL}), \\
& U_{K}(\mathrm{GOAL}) \geq U_{E}(\mathrm{NO} \text { GOAL }) .
\end{aligned}
$$

The result of the above proposition is interesting as it demonstrates that preference differences may mean nothing for how penalty kicks should be executed 
both for Executors and Keepers. It may even come as a surprise to practitioners who seem (in some cases) to believe that Keepers have certain benefits in penalty shoot-outs as opposed to Executors. That may of course be the case from a preference point of view, but as the above proposition 1 indicates, the Nash equilibrium outcome is invariant for the examined game model and preference structure.

\section{Conclusions, model CRiticism And SugGested FurTher Research}

This paper has used various simple game models in order to strengthen the common understanding of the simple yet decisive penalty kick shoot-out sub game in soccer. Several important features have been discussed. The most interesting is perhaps the last point (Section 7) on preference difference invariance. This result is non-trivial from a practitioner's point of view and should, hence, be of interest for practitioners, experts, or fans.

Furthermore, the demonstrated technique of deriving performance data through observing the directional behaviour may seem trivial. However, the potential of this (to the author's knowledge not yet demonstrated technique) is promising. Through more advanced game modelling, one could foresee a simplified technique for gaining performance information as opposed to costly direct observation. The fact that a penalty Executor's abilities to execute penalties may be judged solely on the basis of his choice of shooting direction is a beautiful and perhaps - for certain practitioners - fairly unexpected result.

Finally, the possibility of a unique pure Nash equilibrium outcome, as shown in Figure 2, contrasts much of the existing literature in the field. Though such an outcome (as argued in the paper) might be improbable, the result is interesting from a principal regulator's point of view. As the penalty kick is so decisive in soccer, increased understanding of what mechanisms might change this sub game ought to be of relevance for regulating agents such as UEFA or FIFA.

The models may surely be criticised for their simplicity. After all, applying two player two strategy games of complete information as modelling devices for penalty kicks may seem as too much of a simplification. Many of the arguments given in the paper will be valid if the strategy space is increased and the two-player assumption is perhaps not too hard to accept. However, the inherent assumption of complete information may be of greater importance when the models' ability to spawn reality is considered. Chiappori et al [1] discuss this topic and even try to test such a question empirically. They conclude that incomplete and asymmetric information should not be too much of a problem in penalty kicks. Such a conclusion may be discussed. Surely, penalty kick information is readily available. Through many matches, many Executors and Keepers may be observed and their quality should be observable. Unfortunately, the most decisive situations, the shoot-outs, contain Executors who seldom take penalty kicks. As such, it seems reasonable to accept that the most decisive penalty kicks could contain more asymmetric information than the normal in-game ones. Consequently, moving towards incomplete information formulations may definitely be adequate. Such a task may be an interesting candidate for further research. 


\section{Appendix A. Derivation of equations (6.5)}

The left equation of (6.4) is solved with respect to $\hat{t}_{i}$ yielding

$$
1-\hat{p}_{i}=r_{i}^{*}\left(\hat{t}_{i}-\hat{p}_{i}+1\right) \Rightarrow \hat{t}_{i}=\frac{1-\hat{p}_{i}}{r_{i}^{*}}+\hat{p}_{i}-1 .
$$

Substituting this expression back into the right part of equation (6.4) yields

$$
\begin{aligned}
& \frac{1-\hat{p}_{i}}{r_{i}^{*}}+\hat{p}_{i}-1-\hat{p}_{i}=s_{i}^{*}\left(\frac{1-\hat{p}_{i}}{r_{i}^{*}}+\hat{p}_{i}-1-\hat{p}_{i}+1\right) \\
\Rightarrow & \frac{1-\hat{p}_{i}}{r_{i}^{*}}-1=s_{i}^{*} \cdot \frac{1-\hat{p}_{i}}{r_{i}^{*}}
\end{aligned}
$$

or

$$
\hat{p}_{i}=1-\frac{r_{i}^{*}}{1-s_{i}^{*}} .
$$

Finally, $\hat{t}_{i}$ can be computed by substituting the expression for $\hat{p}_{i}$ from equation (A.2) back into equation (A.1) giving

$$
\begin{aligned}
\hat{t}_{i} & =\frac{1-\left(1-\frac{r_{i}^{*}}{1-s_{i}^{*}}\right)}{r_{i}^{*}}+1-\frac{r_{i}^{*}}{1-s_{i}^{*}}-1 \\
\Rightarrow \hat{t}_{i} & =\frac{\frac{r_{i}^{*}}{1-s_{i}^{*}}}{r_{i}^{*}}-\frac{r_{i}^{*}}{1-s_{i}^{*}}=\frac{1-r_{i}^{*}}{1-s_{i}^{*}} .
\end{aligned}
$$

\section{Appendix B. Proof of proposition 1}

Given the assumptions i) - xi) in Section 4, the added assumption of $q=0$ as well as the preference structure of Table 4 , the normal form game of Figure 3 can be constructed.

In Figure 3, $x, y$ and $z$ are defined as

$$
\begin{aligned}
& x=1+\omega \\
& y=1-\mu \\
& z=(1-p)(1+\omega)+p(1-\mu)
\end{aligned}
$$

Firstly, it is reasonable to check that the best replies (circles and squares in Figure 3) still maintain the structure securing a unique NE in mixed strategies. The best replies for the Executor (circles) are obvious as $1>0$ and $p<1$.

Furthermore, as $1+\omega>1-\mu$ by the given assumptions, the north-west best reply for the Keeper is as indicated in Figure 3. Finally, as

$$
\begin{aligned}
& z>x \Rightarrow(1-p)(1+\mu)+p(1-\mu)>1-\mu \\
\Rightarrow & \omega(1-p)+\mu(1-p)>0
\end{aligned}
$$

obviously holds, the game of Figure 3 has a unique NE in mixed strategies.

To derive this NE, Expected utilities $\left(E_{E}, E_{K}\right.$ for Executor and Keeper respectively) are computed as:

$$
E_{E}=r(1-s)+(1-r) s+(1-r)(1-s) p
$$




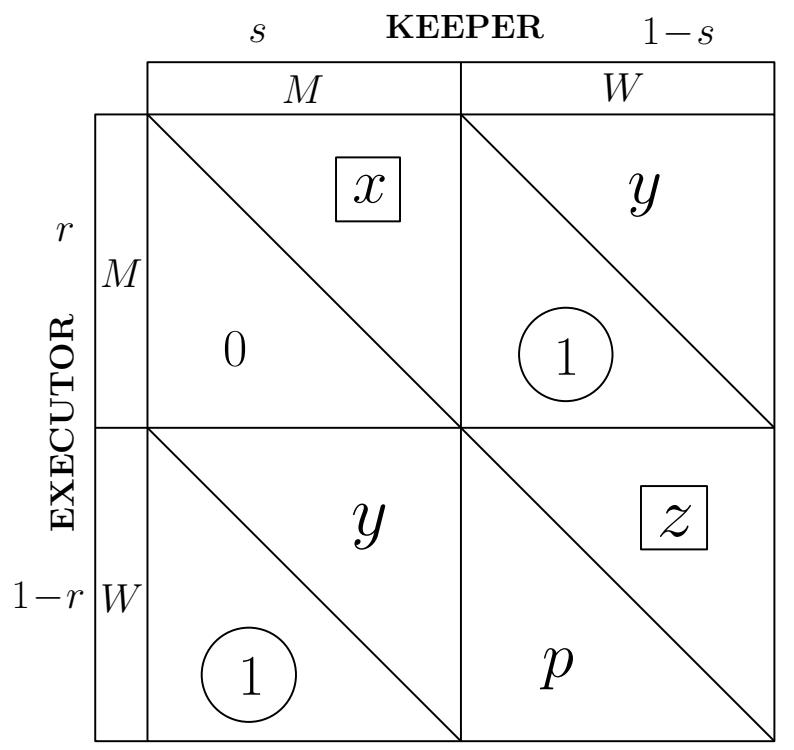

Figure 3. The penalty kick game in normal form with generalized preference differences.

and

$$
E_{K}=r s x+r(1-s) y+(1-r) s y+(1-r)(1-s) z
$$

A necessary condition for this NE is:

$$
\frac{\partial E_{E}}{\partial r}=\frac{\partial E_{K}}{\partial s}=0
$$

giving

$$
\frac{\partial E_{E}}{\partial r}=(1-s)-s-p(1-s)=0 \Rightarrow s^{*}=\frac{p-1}{p-2}
$$

and

$$
\frac{\partial E_{K}}{\partial s}=r x-r y+(1-r) y-z(1-r)=0 \Rightarrow r^{*}=\frac{z-y}{x-2 y+z} .
$$

Finally,

$$
\frac{z-y}{x-2 y+z}=\frac{(1-p)(1+\omega)+p(1-\mu)-(1-\mu)}{(1+\omega)-2(1-\mu)+(1-p)(1+\omega)+p(1-\mu)} .
$$

The numerator $N$ in (B.1) can be expressed as

$$
\begin{aligned}
N & =(1-p)(1+\omega)+p(1-\mu)-(1-\mu) \\
& =(1-p)(1+\omega)+(1-\mu)(p-1)=(1-p)[1+\omega-1+\mu] \\
& =(1-p)(\omega+\mu) .
\end{aligned}
$$


In a similar fashion, the denominator $D$ in equation (B.1) can be expressed

$$
\begin{aligned}
D & =(1+\omega)-2(1-\mu)+(1-p)(1+\omega)+p(1-\mu) \\
& =(1+\omega)[1+1-p]+(1-\mu)[p-2] \\
& =(1+\omega)[2-p]-(1-\mu)[2-p] \\
& =[2-p](1+\omega-1+\mu)=(2-p)(\omega+\mu) .
\end{aligned}
$$

Now, the fraction of equation (B.1) becomes

and finally

$$
\frac{N}{D}=\frac{(1-p)(\omega+\mu)}{(2-p)(\omega+\mu)}=\frac{(1-p)}{(2-p)}=r^{*}
$$

which equals (6.1).

$$
\left\{r^{*}, s^{*}\right\}=\left\{\frac{(1-p)}{(2-p)}, \frac{(1-p)}{(2-p)}\right\}
$$

\section{ReFERENCES}

[1] P. A. Chiappori, S. Levitt and T. Groseclose, Testing mixed-strategy equilibria when players are heterogenous: the case of penalty kicks in soccer, Am. Econ. Rev. 9 (2002), 1138-1151.

[2] M. Bar-Eli and O. H. Azar, Penalty kicks in soccer: An empirical analysis of shooting strategies and goalkeepers preferences, Soccer \& Society 10 (2009), 183-191.

[3] M. Bar-Eli, O. H. Azar, I. Ritov, Y. Keidar-Levin and G. Schein, Action bias among elite soccer goalkeepers: the case of penalty kicks, J. Econ. Psychol. 28 (2007), 606-621.

[4] FIFA, History of the laws of the game, downloaded from: http://www.fifa.com/, FIFA.com, February 2010.

[5] K. K. Haugen, Always Change a Winning Team - a Text on Soccer and Game Theory, Tapir Academic Publishers, Trondheim, 2012.

[6] I. Palacias-Huerta and J. Apestaguia, Psychological pressure in competitive environments: evidence from a randomized natural experiment, Am. Econ. Rev. 100 (2010), 2548-2564.

[7] I. Palacias-Huerta and O. Volij, Experientia docet: professionals play minimax in laboratory experiments, Econometrica 76 (2008), 71-115.

[8] I. Palacias-Huerta, Professionals play minimax, Rev. econ. stud. 70 (2003), 395-415.

[9] G. Jordet, E. Hartman, C. Visscher and K. A. P. M. Lemmink, Kicks from the penalty mark in soccer: the roles of stress, skill, and fatigue for kick outcomes, J. Sport. Sci. (2006), 1-9.

[10] J. K. Leela and D. M. G. Comissiong, Modelling football penalty kicks, Lat. Am. J. Phys. Educ. 3 (2009), 259-269.

[11] J. Nash, Equilibrium points in n-person games, P. Natl. Acad. Sci. USA 36 (1950), 48-49.

Kjetil K. Haugen, Faculty of Economics, Informatics and Social Science, Molde University College, Box 2110, 6402 Molde, Norway

e-mail: kjetil.haugen@himolde.no 